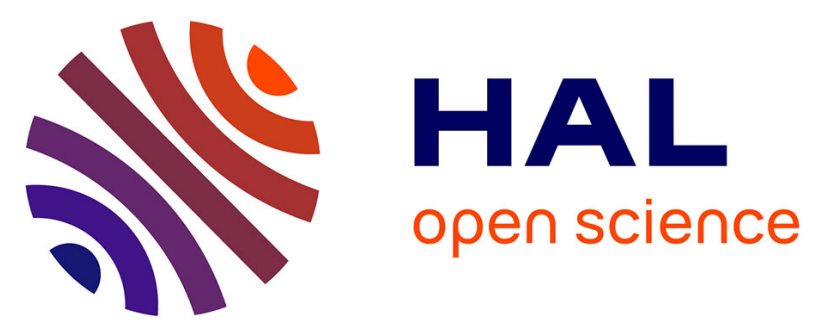

\title{
The Adaptive Cross Approximation Technique for a Volume Integral Equation Method Applied to Nonlinear Magnetostatic Problems
}

Vinh Le-Van, Bertrand Bannwarth, Anthony Carpentier, Olivier Chadebec, Jean-Michel Guichon, Gérard Meunier

\section{To cite this version:}

Vinh Le-Van, Bertrand Bannwarth, Anthony Carpentier, Olivier Chadebec, Jean-Michel Guichon, et al.. The Adaptive Cross Approximation Technique for a Volume Integral Equation Method Applied to Nonlinear Magnetostatic Problems. IEEE Transactions on Magnetics, 2014, 50 (2), pp.445-448. 10.1109/TMAG.2013.2281568 . hal-02277362

\section{HAL Id: hal-02277362 \\ https://hal.science/hal-02277362}

Submitted on 26 Jan 2021

HAL is a multi-disciplinary open access archive for the deposit and dissemination of scientific research documents, whether they are published or not. The documents may come from teaching and research institutions in France or abroad, or from public or private research centers.
L'archive ouverte pluridisciplinaire HAL, est destinée au dépôt et à la diffusion de documents scientifiques de niveau recherche, publiés ou non, émanant des établissements d'enseignement et de recherche français ou étrangers, des laboratoires publics ou privés. 


\title{
The Adaptive Cross Approximation Technique for a Volume Integral Equation Method Applied to Nonlinear Magnetostatic Problems
}

\author{
Vinh Le-Van ${ }^{1}$, Bertrand Bannwarth ${ }^{1}$, Anthony Carpentier ${ }^{1}$, Olivier Chadebec ${ }^{1,2}$, \\ Jean-Michel Guichon ${ }^{1}$, and Gérard Meunier ${ }^{1}$ \\ ${ }^{1}$ Grenoble Electrical Engineering Laboratory, University of Grenoble, Grenoble, France \\ ${ }^{2}$ GRUCAD/EEL/CTC/UFSC, Florianópolis, Brazil
}

\begin{abstract}
Volume integral equation methods are particularly well suited to solve electromagnetic problems, where the air domain is predominant. However, their use leads to the heavy resolution of a dense matrix system. The Adaptive Cross Approximation (ACA) combined with hierarchical matrices $(\mathcal{H}$-matrices) decomposition is an algebraic method allowing the compression of fully populated matrices. This paper presents the ACA technique applied to a volume integral equation to solve nonlinear magnetostatic problems.
\end{abstract}

Index Terms-Adaptive cross approximation, fixed-point method, nonlinear magnetostatic, volume integral equations.

\section{INTRODUCTION}

$\mathbf{V}$ OLUME integral equation methods (VIEM) have the main advantage of avoiding the discretization of inactive regions like the air domain for instance. They are lightweight and accurate methods to compute the far interactions, and can also be applied to the treatment of nonlinear electromagnetic problems. However, the use of the VIEM leads to fully populated matrices because of the integration of the Green's function. Even if an iterative linear solver is used, both the memory and CPU time requirements still increase in a quadratic complexity.

To overcome this limitation, many fast solution methods for integral equations have been intensively developed since the late 1980s, and associated with massively parallel computing resources. The best-known method is obviously the fast multipole method (FMM) [1]. Its multilevel versionthe multilevel FMM [2] is certainly one of the most efficient approaches. However, this type of algorithm is very sequential and although parallel versions can be developed, their performance is sometimes disappointing. The panel clustering method [3], on the same principle of the FMM, gains its efficiency from approximating the Green's function by the Taylor series. The wavelet method [4] reduces significantly the storage needs but does not reduce the computation complexity of the equations system, which is still parabolic.

The ACA combined with $\mathcal{H}$-matrices decomposition [5]-[7] in contrast with previous methods is a purely algebraic method. This approach is easily parallelizable and less intrusive in the source code. In fact, once the method has been developed, its use is independent of the kernel. The ACA was originally introduced in 2000 by M. Bebendorf and since then, has been successfully applied for many integral formulations such as for the boundary element method [6], for the method of moment [8], [9].

The purpose of this paper is to investigate the application of the ACA for a VIEM using the magnetic scalar potential to solve nonlinear magnetostatic problems. Section II is devoted to the presentation of the integral formulation. Then, Section III introduces the resolution of nonlinear problems by the fixed-point method. The outline of the ACA and its implementation technique are the content of Section IV. In Section V, some numerical results that demonstrate the efficiency of the developed method are presented. Finally, this paper concludes in Section VI.

\section{Magnetostatic Volume Integral EQUATion}

For magnetostatic calculations, several integral formulations have already been developed according to the choice of unknowns (magnetization, magnetic field, and induction) and the discretization space. In this paper, the well-known magnetic scalar potential formulation associated to standard node shape functions is chosen. The development of this formulation can be also found in [10].

Let us consider a ferromagnetic material placed in a static magnetic source field, the material region is simply connected and does not contain any current source. The total magnetic scalar potential $\Phi$ at any point of the domain can be represented by

$$
\Phi=\Phi_{0}+\Phi_{\text {red }}
$$

where $\Phi_{0}$ is the magnetic scalar potential of external sources and $\Phi_{\text {red }}$ is the reduced magnetic scalar potential.

The reduced magnetic scalar potential $\Phi_{\text {red }}$ can be expressed as function of the magnetization $\mathbf{M}$, as in

$$
\Phi_{\text {red }}=\frac{1}{4} \int \frac{\mathbf{M} \cdot\left(\mathbf{r}-\mathbf{r}^{\prime}\right)}{\left\|\mathbf{r}-\mathbf{r}^{\prime}\right\|^{3}} d \Omega
$$


where $\Omega$ is the volume of the material; $\mathbf{r}$ and $\mathbf{r}^{\prime}$ are the coordinates of computation and integration points, respectively.

The relation between $\mathbf{M}$ and the total magnetic field $\mathbf{H}$ can be described by

$$
\mathbf{M}=\chi(\mathbf{H}) \mathbf{H}
$$

where $\chi(\mathbf{H})$ is relative magnetic susceptibility of the material.

Because of (3) and the relation $\mathbf{H}=-\nabla \Phi$, substituting $\mathbf{M}$ inside (2) by $-\chi(\mathbf{H}) \nabla \Phi$ and then introducing $\Phi_{\text {red }}$ inside (1), we get the following integral formulation:

$$
\Phi+\frac{1}{4 \pi} \int_{\Omega} \chi(\mathbf{H}) \frac{\nabla \Phi \cdot\left(\mathbf{r}-\mathbf{r}^{\prime}\right)}{\left\|\mathbf{r}-\mathbf{r}^{\prime}\right\|^{3}} d \Omega=\Phi_{0} .
$$

Both collocation and Galerkin's projection methods may be used to solve (4). In fact, a comparison has been performed in [11] and both approaches give comparable results. The collocation is slightly less accurate, but the assemblage of the interaction matrix with collocation method is much faster than with the Galerkin's one. Therefore, the collocation approach is preferred to model the complex applications in this paper.

The magnetic scalar potential is discretized with first-order nodal shape functions and approximated by

$$
\Phi=\sum_{i=1}^{N_{n}} \Phi_{i} \alpha_{i}
$$

where $\Phi_{\mathrm{i}}$ and $\alpha_{i}$ are, respectively, the degree of freedom of $\Phi$ and the first-order nodal shape function associated to the $i$ th mesh node, and $N_{n}$ is the number of mesh nodes.

The collocation approach applied to (4) leads to the following system of algebraic linear equations:

$$
([\mathbf{I}]+[\mathbf{A}]) \boldsymbol{\Phi}=\boldsymbol{\Phi}_{0}
$$

where $[\mathbf{I}]$ is the identity matrix and $[\mathbf{A}]$ is the interaction matrix defined by

$$
A_{i j}=\frac{1}{4 \pi} \int_{\Omega} \chi(\mathbf{H}) \frac{\nabla \alpha_{j} \cdot\left(\mathbf{r}_{i}-\mathbf{r}^{\prime}\right)}{\left\|\mathbf{r}_{i}-\mathbf{r}^{\prime}\right\|^{3}} d \Omega .
$$

A direct or iterative solver could be used to solve (7). Note that the interaction matrix $[\mathbf{A}]$ is a dense matrix of size $N_{n} \times N_{n}$.

\section{NONLINEAR FORMULATION}

The Newton-Raphson (NR) method is a powerful approach to solve a nonlinear system but its application to (4) can be expensive. Indeed, a new computation of the interaction matrix and its derivative is needed at each NR iteration, due to the local change of magnetic susceptibility. So, the fixed point (FP) method can be used to avoid those repeated constructions. In fact, the FP method has been successfully applied to solve nonlinear magnetostatic problems for several integral formulations [12].

In FP method, a given nonlinear characteristic $\mathbf{M}(\mathbf{H})$ can be written as the sum of a linear and nonlinear part, as in

$$
\mathbf{M}(\mathbf{H})=\chi_{\mathrm{FP}} \mathbf{H}+\mathbf{S}
$$

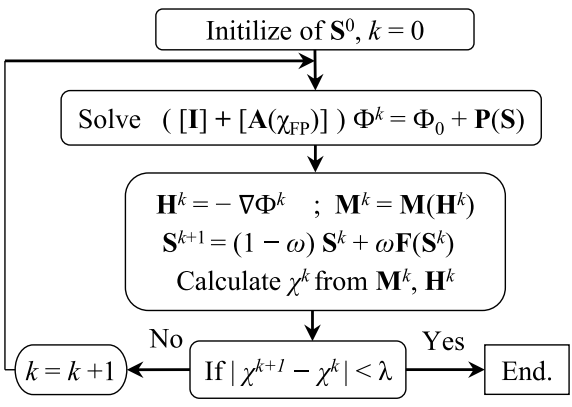

Fig. 1. Algorithm of FP method applied to (14).

which gives rise the following iterative scheme:

$$
\mathbf{S}^{k+1}=\mathbf{M}\left(\mathbf{H}^{k}\right)-\chi_{\mathrm{FP}} \mathbf{H}^{k}=\mathbf{F}\left(\mathbf{S}^{k}\right)
$$

where $\chi_{\mathrm{FP}}$ is the FP constant, $\mathbf{S}$ the nonlinear residual, and $k$ the iteration index. Optimal choice of the FP constant $\chi_{\text {FPopt }}$ can be evaluated like in [13] by

$$
\chi_{\mathrm{FPopt}}=0,5\left(\chi_{\min }+\chi_{\max }\right)
$$

To increase the speed of convergence of FP scheme the iterative scheme (9) may be expressed as

$$
\mathbf{S}^{k+1}=(1-\omega) \mathbf{S}^{k}+\omega \mathbf{F}\left(\mathbf{S}^{k}\right)
$$

where $\omega$ is the relaxation factor, $0<\omega \leq 1$.

Since $\mathbf{H}=-\nabla \Phi$, (8) can be rewritten as in

$$
\mathbf{M}(\mathbf{H})=-\chi_{\mathrm{FP}} \nabla \Phi+\mathbf{S}
$$

Substituting magnetization (12) inside (4) and then introducing $\Phi_{\text {red }}$ inside (1), we get the nonlinear formulation, as in

$\Phi+\frac{1}{4 \pi} \int_{\Omega} \chi_{\mathrm{FP}} \frac{\nabla \Phi \cdot\left(\mathbf{r}-\mathbf{r}^{\prime}\right)}{\left\|\mathbf{r}-\mathbf{r}^{\prime}\right\|^{3}} d \Omega=\Phi_{0}+\frac{1}{4 \pi} \int_{\Omega} \frac{\mathbf{S} \cdot\left(\mathbf{r}-\mathbf{r}^{\prime}\right)}{\left\|\mathbf{r}-\mathbf{r}^{\prime}\right\|^{3}} d \Omega$.

The collocation approach applied on (13) leads to

$$
\left([\mathbf{I}]+\left[\mathbf{A}\left(\chi_{\mathrm{FP}}\right)\right]\right) \boldsymbol{\Phi}=\boldsymbol{\Phi}_{0}+\mathbf{P}(\mathbf{S})
$$

where $\mathbf{P}(\mathbf{S})$ corresponding to the integral term of the right hand side in (13) is the contribution vector of the nonlinear residual.

Briefly, an algorithm to deal with nonlinear systems is shown on Fig. 1 where $\lambda$ is a given accuracy.

\section{ACA TEChNique}

In this section, the principle of the ACA is presented, followed with the hierarchical decomposition of the integral matrices using the $\mathcal{H}$-matrices technique. Section IV-C presents the implementation of compression matrices technique for the nonlinear magnetostatic formulations (14). Note that the detailed analysis as well as the full algorithm of ACA method can be found in [5]-[7]. 


\section{A. Brief Introduction of the ACA}

Let us consider a matrix $\mathbf{M}_{m \times n}$. It can be accurately approximated by a low-rank matrix $\widetilde{\mathbf{M}}_{m \times n}$, which is constructed through a product form. Namely

$$
\mathbf{M}_{m \times n} \approx \widetilde{\mathbf{M}}_{m \times n}=\mathbf{U}_{m \times r} \mathbf{V}_{r \times n}=\sum_{i=1}^{r} \mathbf{u}_{m \times 1}^{i} \mathbf{v}_{1 \times n}^{i}
$$

where $r$ is the rank of $\widetilde{\mathbf{M}}_{m \times n}$ or the effective rank of $\mathbf{M}_{m \times n}$; $\mathbf{U}_{m \times r}$ and $\mathbf{V}_{r \times n}$ are two dense matrices; $\mathbf{u}_{m \times 1}^{i}$ is the column $i$ th of $\mathbf{U}$ and $\mathbf{v}_{1 \times n}^{i}$ is the vector $i$ th of $\mathbf{V}$, respectively. The error of this approximation is controlled by

$$
\left\|\mathbf{M}_{m \times n}-\widetilde{\mathbf{M}}_{m \times n}\right\|_{F} \leq \varepsilon\left\|\mathbf{M}_{m \times n}\right\|_{F}
$$

for a given accuracy $\varepsilon$, where $\left\|\mathbf{M}_{m \times n}\right\|_{\mathrm{F}}$ denotes the Frobenius norm of $\mathbf{M}_{m \times n}$.

The ACA technique is numerically based on the principle of the approximation above but does not need to know all terms of $\mathbf{M}_{m \times n}$. Indeed, only $r$ rows and $r$ columns are calculated during the algorithm. It greatly reduces the number of integral computations to perform. Therefore, not only is the memory requirement reduced but the assembly time is also saved. Yet, if we agree to deal with the product $\mathbf{U}_{m \times r} \mathbf{V}_{r \times n} \mathbf{b}_{n \times 1}$ instead of the exact value $\mathbf{M}_{m \times n} \mathbf{b}_{n \times 1}$, the number of operations needed is remarkably reduced by performing $\mathbf{U}_{m \times r}\left(\mathbf{V}_{r \times n} \mathbf{b}_{n \times 1}\right)$. Thus, if an iterative solver is used, one can save once again the computation time.

One can introduce the compression rate $\eta$ of $\mathbf{M}_{m \times n}$, which denotes the memory saved by using ACA, as in

$$
\eta(\%)=\left(1-\frac{r \cdot(m+n)}{m \cdot n}\right) \cdot 100 \% .
$$

Obviously, the approximation by ACA approach above is only useful if $r<(1 / 2) \min \{m, n\}$, namely $\mathbf{M}_{m \times n}$ presents similar values. With VIEM, this only happens if the integration kernel is asymptotically smooth and the matrix deals with sufficiently far interactions. Thus, for a given mesh, it is necessary to use a particular technique to generate a block partitioning of the integration matrix.

\section{B. Hierarchical Decomposition of the Integration Matrix}

It is well known that large dense matrices arising from integral equations have no explicit structure in general. As a rule, these matrices cannot be represented by a low-rank approximation. However, it is possible to decompose these matrices into a hierarchical system of blocks, which contains rather large blocks representing the interaction between two well-separated groups, thanks to $\mathcal{H}$-matrices technique.

To be efficient, the block matrices to compress must involve interactions between distant subspaces where the integration kernel is sufficiently smooth. With the help of an octree partition of the space, the degrees of freedom in the mesh can be renumbered to fulfil this criterion. The goal is to slip the domain into subdomains satisfying the admissibility condition:

$$
\operatorname{diam}(\sigma)+\operatorname{diam}(\tau) \leq 2 \text { dist }(\sigma, \tau)
$$

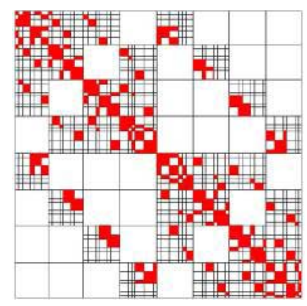

Fig. 2. Example of $\mathcal{H}$-matrices decomposition.

where $\sigma$ is the source cube, $\tau$ is the target cube; $\operatorname{diam}($. denotes diameter of bounding spheres, and $\operatorname{dist}(\sigma, \tau)$ the distance between two cubes.

Based on the admissibility condition, a hierarchical block structure representation of matrices is obtained, and the interactions between groups are treated by either near or far interaction. The near interactions will be evaluated exactly by a full matrix computation. The far interactions will be compressed by ACA.

The hierarchical block partitioning above is a part of the $\mathcal{H}$-matrices technique whose formal definition and description is in [14]. In Fig. 2, an example of the $\mathcal{H}$-matrices decomposition of matrix $\left[\mathbf{A}\left(\chi_{\mathrm{FP}}\right)\right]$ is shown. There, the red blocks represent the near interactions and the white ones represent the far interactions.

\section{Implementation of the Compression Matrices Technique}

We summarize, the implementation of the ACA technique for the nonlinear magnetostatic formulation using scalar magnetic potential in (14). Note that the assembly of vector $\mathbf{P}(\mathbf{S})$ in (14) can be decomposed into a product, as in

$$
\mathbf{P}(\mathbf{S})=\left[\left[\mathbf{G}_{x}\right]+\left[\mathbf{G}_{y}\right]+\left[\mathbf{G}_{z}\right]\right]\left(\begin{array}{c}
\mathbf{S}_{\mathbf{x}} \\
\mathbf{S}_{\mathbf{y}} \\
\mathbf{S}_{\mathbf{z}}
\end{array}\right)
$$

where $\left[\mathbf{G}_{\mathrm{x}}\right],\left[\mathbf{G}_{\mathrm{y}}\right]$, and $\left[\mathbf{G}_{\mathrm{z}}\right]$ of size $N_{n} \times N_{e}$ with $N_{e}$ the number of mesh elements are assembly component matrices of the derivative Green kernel; and $\mathbf{S}_{\mathbf{x}}, \mathbf{S}_{\mathbf{y}}$, and $\mathbf{S}_{\mathbf{z}}$ are the three components of $\mathbf{S}$. Thus, $\left[\mathbf{G}_{\mathrm{x}}\right],\left[\mathbf{G}_{\mathrm{y}}\right]$, and $\left[\mathbf{G}_{\mathrm{z}}\right]$ as well as $\left[\mathbf{A}\left(\chi_{\mathrm{FP}}\right)\right]$ are built only once and depend on the mesh geometry. These four matrices are compressed thanks to the ACA combined with $\mathcal{H}$-matrices decomposition. Finally, to solve the linear equation (14) with the compressed matrices, the iterative solver GMRes is chosen.

\section{Numerical Result}

The proposed procedure has been applied to the analysis of an academic test case. Let us consider a coil contactor-like problem. Its geometry is described in Fig. 3(a). The nonlinear property of ferromagnetic material of the contactor is given in Fig. 3(b). The magnetic region is discretized by tetrahedral elements. The source coil is $3000 \mathrm{AT}$ and the scalar potential $\Phi_{0}$ is computed with a finite element method (FEM) [11]. The accuracy $\varepsilon$ of the ACA algorithm is set to $10^{-4}$. All the computations were performed on a personal computer with Intel Core i5 $2.2 \mathrm{GHz} \times 64 \mathrm{CPU}$ and $4 \mathrm{~GB}$ of RAM.

The combination of the VIEM with the ACA allows us to increase the maximal limit of the number of mesh elements. 


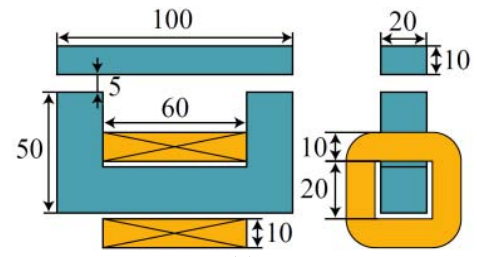

(a)

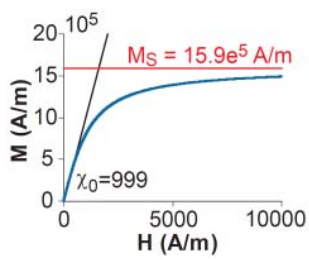

(b)
Fig. 3. Contactor problem. (a) Geometry. (b) Behavior law.

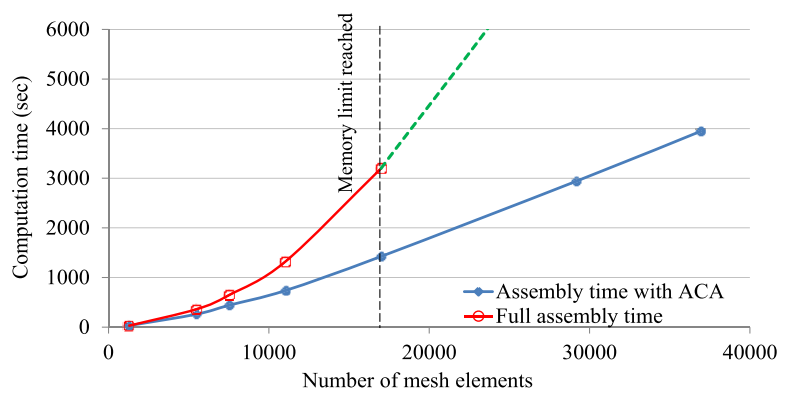

Fig. 4. Evolution of computation times.

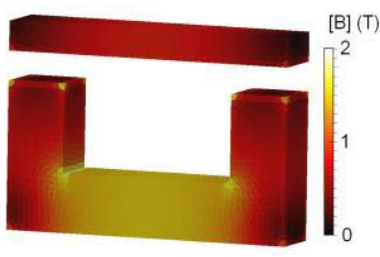

(a)

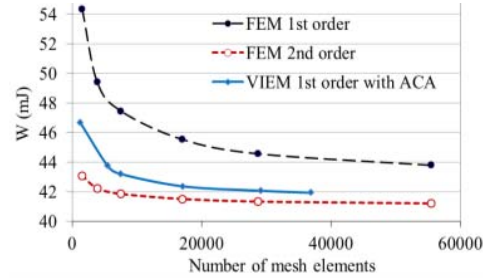

(b)
Fig. 5. Resolution. (a) Distribution of B. (b) Comparison result with FEM.

Namely, by using the coupling the VIEM and the ACA, one can solve the problem with a mesh of 37000 elements whereas if used the pure VIEM (without ACA) this one is limited at 17000 elements. Besides, the resolution is also faster because the complexity of the matrix-vector product has been decreased. However, for a 17000 elements mesh, the resolution time is only reduced from 195 to $165 \mathrm{~s}$, so the gain is not so important. This can be explained by the fact that there are too many block generated and that the compression rate of the involved matrix $([\mathbf{A}])$ is smaller.

The evolution of the computation times for the assembly with both approaches (full and used $\mathcal{H}$-matrices) with respect to the number of mesh elements are shown in Fig. 4. It shows that the $\mathcal{H}$-matrices assembly has an evolution better than $O\left(N_{e}^{2}\right)$, close to $O\left(N_{e} \log \left(N_{e}\right)\right)$.

Fig. 5(a) shows the distribution of the magnetic field $\mathbf{B}$ for a mesh of 37000 tetrahedral elements. To solve (14) with this mesh, only $1.9 \mathrm{~GB}$ of RAM is required for the assembly of the matrices $\left[\mathbf{A}\left(\chi_{\mathrm{FP}}\right)\right],\left[\mathbf{G}_{\mathrm{x}}\right],\left[\mathbf{G}_{\mathrm{y}}\right]$, and $\left[\mathbf{G}_{\mathrm{z}}\right]$. Without compression, $8.3 \mathrm{~GB}$ of RAM would be necessary, thus the memory need is decreased by $77 \%$ with the proposed procedure. By using the fixed-point method with $\chi_{\mathrm{FP}}$ as 650 and $\omega$ as 1 , the nonlinear resolution converged after 158 iterations with $6 \mathrm{~s}$ for each iteration time.
The result of the developed method has been compared with the FEM thanks to the software FLUX. Fig. 5(b) shows the evolution of the total magnetic energy of the contactor with respect to the number of mesh elements. In fact, we solved by FEM with both first- and second-order magnetic scalar potential formulations, respectively. For the coupling of VIEM and ACA, the first-order elements are used. Obviously, the three results converged to the same value. The result obtained by developed method is highly accurate and very close to the FEM second order.

\section{CONCLUSION}

The ACA combined with $\mathcal{H}$-matrices has been applied to a nonlinear magnetostatic problem with a VIEM using the magnetic scalar potential. The results are significant to the treatment of fine meshes with a gain of memory and computation time. To improve the use of the $\mathcal{H}$-matrices, a decomposition technique based on binary trees instead of an octree is envisaged to reduce the number of needed blocks. This work on the $\mathcal{H}$-matrices arithmetic will be performed to allow better preconditioning techniques.

\section{REFERENCES}

[1] L. Greengard and V. Rokhlin, "A fast algorithm for particle simulations," J. Comput. Phys., vol. 73, no. 2, pp. 325-348, 1987.

[2] J. Carrier, L. Greengard, and V. Rokhlin, "A fast adaptive multipole algorithm for particle simulations," SIAM J. Sci. Statist. Comput., vol. 9, no. 4, pp. 669-686, 1988.

[3] W. Hackbusch and Z. P. Nowak, "On the fast matrix multiplication in the boundary element method by panel clustering," Numer. Math., vol. 54, no. 4, pp. 463-491, 1989.

[4] G. Beylkin, R. Coifman, and V. Rokhlin, "Fast wavelet transforms and numerical algorithms. I," Commun. Pure Appl. Math., vol. 44, no. 2, pp. 141-183, 1991.

[5] M. Bebendorf, "Approximation of boundary element matrices," Numer. Math., vol. 86, no. 4, pp. 565-589, Jun. 2000.

[6] S. Kurz, O. Rain, and S. Rjasanow, "The adaptive cross-approximation technique for the 3-D boundary element method," IEEE Trans. Magn., vol. 38, no. 2, pp. 421-424, Mar. 2002.

[7] M. Bebendorf and S. Rjasanow, "Adaptive low-rank approximation of collocation matrices," Computing, vol. 70, no. 1, pp. 1-24, Mar. 2003.

[8] Y. Takahashi, C. Matsumoto, and S. Wakao, "Large-scale and fast nonlinear magnetostatic field analysis by the magnetic moment method with the adaptive cross approximation," IEEE Trans. Magn., vol. 43, no. 4, pp. 1277-1280, Apr. 2007.

[9] K. Zhao, M. N. Vouvakis, and J.-F. Lee, "The adaptive cross approximation algorithm for accelerated method of moments computations of EMC problems," IEEE Trans. Electromagn. Comput., vol. 47, no. 4, pp. 763-773, Nov. 2005.

[10] A. G. Kalimov, "Three-dimensional magnetostatic field calculation using integro-differential equation for scalar potential," IEEE Trans. Magn., vol. 32, no. 3, pp. 667-670, May 1996.

[11] A. Carpentier, O. Chadebec, N. Galopin, G. Meunier, and B. Bannwarth, "Resolution of nonlinear magnetostatic problems with a volume integral method using the magnetic scalar potential," IEEE Trans. Magn., vol. 49, no. 5, pp. 1685-1688, May 2013.

[12] A. Canova and M. Repetto, "Integral solution of nonlinear magnetostatic field problems," IEEE Trans. Magn., vol. 37, no. 3, pp. 1070-1077, May 2001.

[13] M. Chiampi, A. Negro, and M. Tartaglia, "A finite element method to compute three-dimensional field distribution in transformer cores," IEEE Trans. Magn., vol. 16, no. 6, pp. 1413-1419, Nov. 1980.

[14] W. Hackbusch, "A sparse matrix arithmetic based on H-matrices. I. Introduction to H-matrices," Computing, vol. 62, no. 2, pp. 89-108, 1999. 